\title{
ASTEROIDS IN GALEX: NEAR-ULTRAVIOLET PHOTOMETRY OF THE MAJOR TAXONOMIC GROUPS
}

\author{
Adam WaszczaK ${ }^{1}$, Eran O. OfeK ${ }^{2,3}$, and Shrinivas R. KulKarni ${ }^{1}$ \\ ${ }^{1}$ Division of Geological and Planetary Sciences, California Institute of Technology, Pasadena, CA 91125, USA; waszczak@caltech.edu \\ ${ }^{2}$ Benoziyo Center for Astrophysics, Faculty of Physics, Weizmann Institute of Science, Rehovot 76100, Israel \\ ${ }^{3}$ Helen Kimmel Center for Planetary Science, Weizmann Institute of Science, Rehovot 76100, Israel \\ Received 2015 April 16; accepted 2015 May 6; published 2015 August 13
}

\begin{abstract}
We present ultraviolet (UV) photometry (near-UV (NUV) band, 180-280 nm) of 405 asteroids observed serendipitously by GALEX from 2003 to 2012 . All asteroids in this sample were detected by GALEX at least twice. Unambiguous visible-color-based taxonomic labels (C type versus $\mathrm{S}$ type) exist for 315 of these asteroids; of these, thermal-infrared-based diameters are available for 245 . We derive NUV - $V$ color using two independent models to predict the visual magnitude $V$ at each NUV-detection epoch. Both $V$ models produce NUV $-V$ distributions in which the $\mathrm{S}$ types are redder than $\mathrm{C}$ types with more than $8 \sigma$ confidence. This confirms that the $\mathrm{S}$ types' redder spectral slopes in the visible remain redder than the C types' into the NUV, this redness being consistent with absorption by silica-containing rocks. The GALEX asteroid data confirm earlier results from the International Ultraviolet Explorer, which two decades ago produced the only other sizeable set of UV asteroid photometry. The GALEX-derived NUV - V data also agree with previously published Hubble Space Telescope (HST) UV observations of asteroids 21 Lutetia and 1 Ceres. Both the HST and GALEX data indicate that NUV band is less useful than $u$ band for distinguishing subgroups within the greater population of visible-color-defined $\mathrm{C}$ types (notably, M types and G types).
\end{abstract}

Key words: minor planets, asteroids: general - surveys

Supporting material: machine-readable table

\section{INTRODUCTION}

As in visible wavelengths, ultraviolet (UV) flux from asteroids is reflected sunlight. However, the steep drop in the solar spectrum shortward of $\sim 300 \mathrm{~nm}$ (Figure 1) makes asteroids orders of magnitude fainter in the UV than in the visible. For this reason-as well as the strong UV absorption by atmospheric ozone-UV observations of asteroids typically employ the Hubble Space Telescope (HST) or specialized instruments on a space-mission payload physically closer to the asteroid. These constraints have generally prohibited largesample demographic studies of asteroids in the UV.

Predating HST, the International Ultraviolet Explorer (IUE) targeted 45 asteroids from 1978 to 1992, producing what remains to date the largest published sample of near-UV (NUV) asteroid spectra (Roettger \& Buratti 1994), specifically in the range of $230-325 \mathrm{~nm}$. The IUE data show evidence of clustering, principally with respect to UV geometric albedo. This clustering becomes further evident when coarsely defined visible spectral type is included as a categorical parameter for each object $(\mathrm{C}, \mathrm{S}$ and $\mathrm{M}$ types being the classes considered in the original work). Comparing the IUE-derived UV geometric albedos for each class with the visible light geometric albedos demonstrated that the $\mathrm{S}$ types, which are redder-colored in the visible (specifically, $400-800 \mathrm{~nm}$ ), remain redder than C types into the NUV.

The IUE data, combined with previously measured visible spectra, suggested that asteroid reflectances over the entire NUV to visible wavelength range $(200-800 \mathrm{~nm})$ are generally consistent with those of silica-bearing rocks (e.g., Wagner et al. 1987). To first order, this trend is characterized by an increase in a rock's reflectance at longer wavelengths, generally attributable to the decreased fraction of volume-scattered light, i.e., light which penetrates into the mineral grains. The intensity of volume-scattered light varies as $\exp (-k d / \lambda)$, where $d$ is the grain size, $k$ is the imaginary part of the index of refraction, and $\lambda$ is the wavelength. Once refracted into the grains, volumescattered light is subject to absorption by various ( $\lambda$ dependent) interactions with the mineral's crystalline structure. At sufficiently short wavelengths (i.e., well into the UV region), most incident light penetrates the grains and is absorbed, while the small amount of measured reflected light is predominantly scattered directly from the grain surface. Precise characterization of this transition between surfacedominated and surface plus volume-scattered reflectance-as well as the identification of any additional mineral-specific spectral features-is therefore useful for tying astronomical observations of asteroids to laboratory-measured analogs, including lunar and meteoritic samples.

In this work we aim to verify the IUE's findings with a newer and larger sample of UV asteroid data from the GALEX, a NASA Small Explorer-class space telescope mission which from 2003 to 2012 conducted a UV imaging survey in a far-UV band (FUV, 130-190 nm) and a NUV band (180-280 nm). Approximately $2 / 3$ of the sky was covered, with avoidance of bright stars and low galactic latitudes. Martin et al. (2005) discuss the extragalactic science program, while Morissey et al. $(2005,2007)$ discuss the on-orbit performance, survey calibration and data products. GALEX has a $50 \mathrm{~cm}^{2}$ effective area, 1:25 diameter circular field of view, and FWHM resolution of 4!.5 in the NUV. Programs within the GALEX mission included an all-sky survey (AIS, with $\sim 100 \mathrm{~s}$ exposures) and a medium-depth survey (MIS, with $\sim 1500 \mathrm{~s}$ exposures), and also a spectroscopic (grism) survey. Figure 1 shows the photometric response functions of the two GALEX bandpasses multiplied by the solar spectrum, with comparison to the ugriz visible bandpasses. Detection of asteroids in the FUV is extremely unlikely (nonetheless, as described below we searched for both NUV and FUV asteroid detections). 


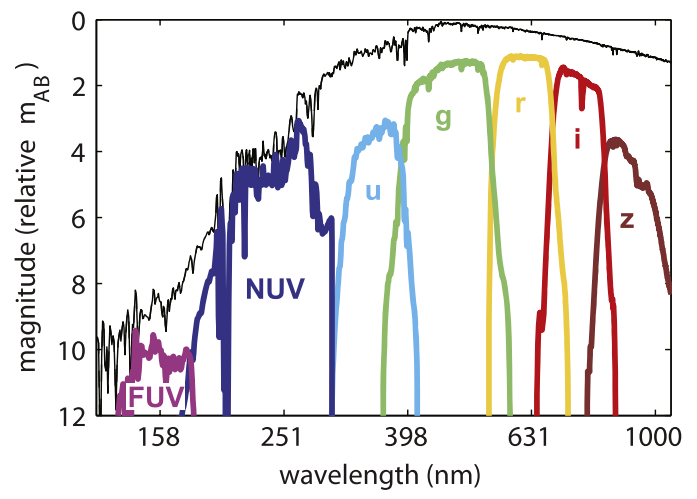

Figure 1. GALEX UV and SDSS visible filter response curves (colored lines) multiplied by the spectrum of a G2 V type star (black line). The spectrum is from the library of Pickles (1998). The vertical scale is in AB magnitude units per unit wavelength, offset by an arbitrary constant. Note wavelength is plotted on a log scale.

Our approach in analyzing GALEX asteroid observations differs somewhat from Roettger \& Buratti's treatment of the IUE data. First, instead of referencing taxonomic class labels (e.g., "C type," "S type," "M type," etc.) assigned to individual asteroids by previous authors, we define classes using a color index derived from a clustering analysis performed on a compilation of seven visible-color surveys (Waszczak et al. 2015). The brightest asteroids typically were targeted in one or more spectroscopic surveys-e.g., the Eight Color Asteroid Survey (ECAS, Zellner et al. 1998) or the Small Main-Belt Asteroid Spectroscopic Surveys (SMASS; Xu et al. 1995; Bus \& Binzel 2002). Dimmer objects however often only have color information from the Sloan Digital Sky Survey (SDSS; York et al. 2000; Ivezić et al. 2001; Parker et al. 2008). The color index of Waszczak et al. (2015) puts asteroids of all sizes on a single, quantitative color scale (a proxy for spectral slope), the endmembers of which we identify with the C-type and S-type complexes. We use the terms " $\mathrm{C}$ types" and "S types" purely for compatibility with the literature, noting that our color index combined with the classification thresholds we apply to it represent original definitions of these two groups.

Use of a one-dimensional color metric sacrifices sensitivity to secondary taxonomic groups such as $\mathrm{M}$ types, $\mathrm{D}$ types and $\mathrm{V}$ types. While our purpose is mainly to examine the first-order taxonomic variation, in certain cases we divide our colordefined "C types" into two classes $\left(\mathrm{C}_{\text {high }}\right.$ and $\left.\mathrm{C}_{\text {low }}\right)$ on the basis of visible and near-infrared albedo (as detailed in Section 3.2). At the end of this work we also examine well-established C-complex subgroups from both the Tholen and Bus/Binzel taxonomies (Section 6), showing how these known subgroups (e.g., $M$ types and $\mathrm{G}$ types) are distributed in this work's coloralbedo space and highlighting these subgroups' unique UV properties.

A second distinction between this work's approach and Roettger \& Buratti (1994) is that, rather than comparing the geometric albedo in the UV with that of the visible band, we focus on the difference in apparent magnitudes between the $\mathrm{UV}$ and visible. One motivation for doing this is we need not make any assumptions about the phase function of asteroids in the UV. The challenge however is that we must accurately estimate the visible flux at the time of the UV observations. As discussed in Section 3, we adopt (and compare) two distinct methods for predicting the visual magnitude. The first method simply adopts the widely used MPC ${ }^{4}$ predicted magnitudes; the second method applies color-dependent phase-function and bond-albedo estimates adapted from the Waszczak et al. (2015) study of lightcurves from the Palomar Transient Factory survey $^{5}$ (PTF; Law et al. 2009; Rau et al. 2009).

\section{GALEX ASTEROID OBSERVATIONS}

Extracting detections of known asteroids from a survey involves a three-dimensional (R.A., decl., time) cross-matching of ephemerides against the survey's time-stamped image boundaries (e.g., Ofek 2012). We modified software originally used to search for asteroids in PTF (Waszczak et al. 2013, 2015) to instead search for asteroids in GALEX.

We first retrieved the metadata of all GALEX images, available from the Space Telescope Science Institute via command-line queries with the CasJobs tool (Li \& Thakar 2008). We then indexed all image centers with respect to (R.A., decl.) into uniformly spaced sky cells of $3^{\circ}$ radius. For all $\sim 380,000$ numbered asteroids, we queried JPL's online service HORIZONS (Giorgini et al. 1996) to generate a 1-day-spaced ephemeris spanning 2003-2012. Using an object-specific search radius equal to $3^{\circ}$ (cell radius) plus 0.75 (FOV radius) plus the object's maximum 1-day motion $(\sim 10$ arcmin for most main-belt objects), we matched the ephemeris points against the sky cells. For each matched cell, we filtered out all images in that cell not within the epoch range of the matched ephemeris points, then for each surviving image we re-queried HORIZONS for the precise location at each observed epoch. We next performed a 1:25radial match of these precise positions against the relevant GALEX image centers.

We found $\sim 850,000$ predicted detections of numbered asteroids (with no limit on apparent magnitude) in GALEX using this method. For each predicted detection, using CasJobs we queried the GALEX single-visit source list (as opposed to the co-added source list). Multiple matches near the same point occurring more than $6 \mathrm{hr}$ apart were excluded, as were all matches further than $2^{\prime \prime}$ from the predicted location. Additionally, to ensure the inclusion of greater than (approximately) $5 \sigma$ detections, we discarded all matches with NUV $>21 \mathrm{mag}$ in the shorter exposures (AIS program), and discarded all matches with NUV > 22.7 mag in the longer exposures (MIS program), following the limiting magnitudes quoted by Morissey et al. (2007).

Following the above procedure and criteria, we extracted a total of 1342 positive NUV detections of 405 unique asteroids which were detected by GALEX at least twice (and no FUV detections, as expected). These detections are listed in Table 1; several histograms detailing these detections appear in Figure 2.

All of the GALEX-observed asteroids are in the main-belt; the sample includes no near-Earth or outer-solar system objects.

\section{MODELING VISIBLE MAGNITUDES}

In this section we consider two distinct methods of estimating the visible magnitudes corresponding to all GALEX NUV detections; this in turn provides the distribution of the asteroids' NUV - $V$ color. The general model for an asteroid's

\footnotetext{
4 IAU Minor Planet Center, http://minorplanetcenter.net.

5 http://ptf.caltech.edu
} 
Table 1

Observations of Asteroids Detected in GALEX NUV Images

\begin{tabular}{|c|c|c|c|c|c|c|c|c|c|}
\hline $\begin{array}{l}\text { Asteroid } \\
\text { Number } \\
\end{array}$ & $\begin{array}{l}\text { Observation } \\
\text { Date (UT) }\end{array}$ & $\begin{array}{l}\text { Detected } \\
\text { R.A. (deg) }\end{array}$ & $\begin{array}{c}\text { Detected } \\
\text { decl. (deg) }\end{array}$ & $\begin{array}{c}\text { Position } \\
\text { Residual (") }\end{array}$ & $\begin{array}{l}\text { NUV } \\
\text { Mag }\end{array}$ & $\begin{array}{l}\text { NUV Mag } \\
\text { Uncertainty }\end{array}$ & $\begin{array}{c}\text { MPC-predicted } \\
\text { Visible Mag }\left(V_{\mathrm{MPC}}\right) \\
\end{array}$ & $\begin{array}{l}\text { Exposure } \\
\text { Time }(\mathrm{s}) \\
\end{array}$ & $\begin{array}{c}\text { Unique Database ID } \\
\text { (obj ID Key in CasJobs) }\end{array}$ \\
\hline 1 & 2011 Oct 12.65797 & 355.22180 & -18.47266 & 0.5 & 14.38 & 0.01 & 8.0 & 91 & 6380556162844065792 \\
\hline 1 & 2011 Oct 21.42095 & 353.95617 & -18.39556 & 0.2 & 14.67 & 0.01 & 8.1 & 80 & 6380556163951362048 \\
\hline 3 & 2005 Dec 26.72536 & 75.12144 & -1.31299 & 1.4 & 14.43 & 0.01 & 7.8 & 80 & 6381858059773280256 \\
\hline 3 & 2011 Apr 17.04995 & 166.80140 & 8.52138 & 1.4 & 16.30 & 0.01 & 9.8 & 1513 & 3855329770719936512 \\
\hline 6 & 2006 Aug 29.56208 & 309.25038 & -19.31464 & 0.9 & 14.78 & 0.01 & 8.4 & 112 & 6379782093773209600 \\
\hline 6 & 2005 May 7.46079 & 204.16985 & 11.58510 & 0.4 & 16.67 & 0.02 & 10.2 & 112 & 6378656257217134592 \\
\hline 8 & 2004 Dec 21.41462 & 122.52225 & 19.17684 & 0.7 & 15.63 & 0.02 & 9.0 & 92 & 6377776615736213504 \\
\hline 8 & 2004 Dec 21.48313 & 122.51009 & 19.18345 & 0.1 & 15.60 & 0.02 & 9.0 & 87 & 6377776615769767936 \\
\hline
\end{tabular}

Note. Includes 1342 detections of 405 asteroids detected at least twice.

(This table is available in its entirety in machine-readable form.) 

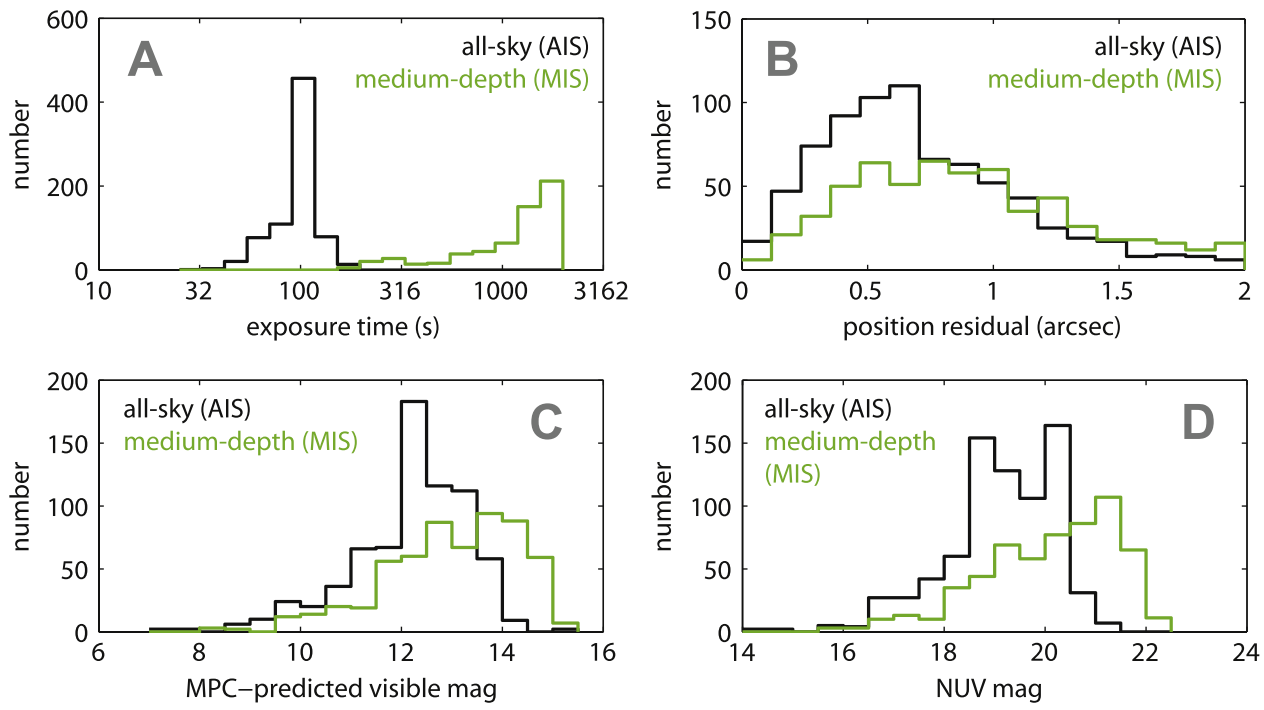

Figure 2. Characteristics of positive asteroid detections from the two GALEX surveys (distinguished by exposure time) shown separately in black and green.

apparent visual magnitude $V(\log$ flux $)$ is

$$
V=H+\delta+5 \log _{10}(r \Delta)-2.5 \log _{10}[\phi(\alpha)] \text {, }
$$

where $H$ is the absolute magnitude (a constant), $\delta$ is a periodic variability term due to rotation (e.g., if the object is spinning and has some asymmetry in shape or albedo), $r$ and $\Delta$ are the heliocentric and geocentric distances (in AU), and $\phi=\phi(\alpha)$ is the phase function, which varies with the solar phase angle $\alpha$ (the Sun-asteroid-Earth angle). When $\alpha=0$ (i.e., at opposition), $\phi=1$ by definition, while in general $0<\phi<1$ for $\alpha>0$ (with $\phi$ decreasing as $\alpha$ increases).

All asteroids for which we have extracted GALEX observations have known orbits, meaning $r, \Delta$, and $\alpha$ are accurately and precisely known at all observed epochs. Our two methods for estimating $V$ differ in their assumptions regarding (and observational data used to constrain) $H$ and $\phi$. In both cases we do not attempt to model the rotational term $\delta$, but rather incorporate $\delta$ into the uncertainty of $V$ using lightcurve amplitude estimates from the literature. In particular, 388 of the 405 GALEX-observed asteroids have an amplitude lowerlimit estimate available in the Lightcurve Database (Warner et al. 2009; Harris et al. 2012).

In the following sections we refer to two different albedo quantities. The visible-band geometric albedo $p_{V}$ relates to the visible-band bond albedo $A_{\text {bond }}$ and the phase function $\phi$ (of Equation (1)) according to

$$
p_{V} \equiv \frac{A_{\text {bond }}}{2}\left(\int_{0}^{\pi} \phi(\alpha) \sin (\alpha) d \alpha\right)^{-1} \equiv \frac{A_{\text {bond }}}{q} \text {, }
$$

The above equation also defines the phase integral $q$. The bond albedo $A_{\text {bond }}$ is defined as the total visible light energy reflected or scattered by the asteroid (in all directions) divided by the total visible light energy incident upon the asteroid (from the Sun). Assuming the asteroid has a circular cross-section of diameter $D$, this can be expressed as

$$
A_{\text {bond }} \equiv \frac{\int_{0}^{\pi} f(\alpha) \sin (\alpha) d \alpha}{\left(f_{\text {Sun }} / 4 \pi \mathrm{AU}^{2}\right) \times \pi(D / 2)^{2}},
$$

where $f(\alpha)=10^{-V(\alpha) / 2.5}$ is the asteroid's flux as a function of phase angle, with $V(\alpha)=H-2.5 \log _{10} \phi(\alpha)$ being Equation (1) evaluated at $\delta=0$ and $r=\Delta=1$ AU (similarly, $\left.f_{\text {Sun }}=10^{-V_{\text {Sun }} / 2.5}\right)$.

\section{1. (H, G) from MPC Data}

The first method for estimating $V$ adopts the Minor Planet Center's computed absolute magnitudes $\left(H_{\mathrm{MPC}}\right)$, which are regularly updated by the MPC's automated processes and utilize the Lumme-Bowell $G$-parameter model for $\phi$ (Bowell et al. 1989, pp. 524-556). This same $(H, G)$ model then predicts the apparent magnitude $V_{\mathrm{MPC}}$ as a function of solar phase angle.

The $H_{\text {MPC }}$ values are fit to photometry provided by a variety of surveys/individuals, many of whom may use slightly different absolute calibration standards or filters with slightly different specifications. A small fraction of asteroids have fitted $G$ values; Harris \& Young (1988) present mean $G$ values for several major taxonomic classes, with $G=0.15$ being an average between the $\mathrm{C}$ types $(G \approx 0.08)$ and the $\mathrm{S}$ types $(G \approx 0.23)$. For the majority of asteroids the MPC uses an assumed $G=0.15$ with this model. Waszczak et al. (2015) compares the $H_{\mathrm{MPC}}$ values with $H$ magnitudes derived from a model that includes rotation and the more modern $\left(H, G_{12}\right)$ phase function of Muinonen et al. (2010). Among bright asteroids the relative difference is typically between $0.3 \%$ and $3 \%$, corresponding to (on average) an $\sim 0.07$ mag discrepancy.

Though $H_{\text {MPC }}$ values are available for all 405 GALEXobserved asteroids, we only consider the subset of 315 asteroids having visible-band color indices of either less than 0.25 ("C types") or greater than 0.75 ("S types"). Of these, 41 asteroids have $G_{\mathrm{MPC}} \neq 0.15$. 

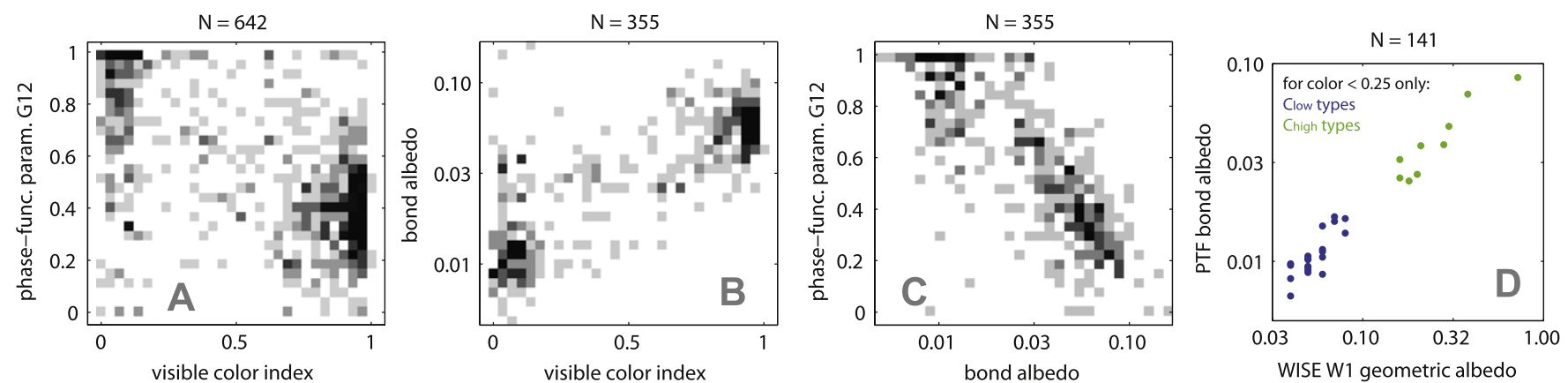

Figure 3. We compute $V_{\mathrm{PTF}}$ model magnitudes by first assigning fixed $A_{\mathrm{bond}}$ and $G_{12}$ values to each $G A L E X$-observed asteroid depending on its color class; we then use $D$ to compute $H$, and finally use the assumed $G_{12}$ value to predict $V$. The fixed values of $A_{\text {bond }}$ and $G_{12}$ are medians from the color-albedo- $G_{12}$ data in Waszczak et al. (2015), 2D histograms of which are shown here. Above each plot is the sample size $(N=\ldots)$. A total of 642 asteroids have color data and $G_{12}$ values in the PTF data; 355 of these also have diameters available (required to compute $A_{\text {bond }}$ ). Panel (D) shows that WISE W1 geometric albedos correlate with the PTF bond albedo; we thus use the WISE $p_{W 1}$ data to assign $\mathrm{C}$ types either a low $\left(A_{\text {bond }} \approx 0.01\right)$ or high $\left(A_{\text {bond }} \approx 0.04\right)$ bond albedo.

\section{2. $\left(D, A_{\text {bond }}, G_{12}\right)$ from PTF, Infrared, and Color Data}

Our second means of estimating visual magnitudes applies only to asteroids having both a color index and a diameter estimate constrained from thermal fluxes in an infrared survey. ${ }^{6}$ In this approach we use the $G_{12}$-parameter model for $\phi$ (Muinonen et al. 2010), and we replace $H$ with its equivalent expression $^{7}$ in terms of the diameter $D$, bond albedo $A_{\text {bond }}$, and phase integral $q$ :

$$
H=-5 \log _{10}\left(\frac{D \sqrt{A_{\text {bond }} / q}}{1329 \mathrm{~km}}\right),
$$

where the phase integral $q$ is a linear function of $G_{12}$ :

$$
q\left(G_{12}\right)= \begin{cases}0.2707-0.236 G_{12} & \text { if } G_{12}<0.2 \\ 0.2344-0.054 G_{12} & \text { otherwise }\end{cases}
$$

We again define "C types" as all asteroids with color indices less than 0.25 and "S types" as all with color indices greater than 0.75 . For $\mathrm{S}$ types we then consider diameters derived from any of four infrared surveys (see Footnote 6), while for $\mathrm{C}$ types we specifically require that the asteroid have been observed in the WISE 4-band cryogenic survey (Wright et al. 2010; Masiero et al. 2014 and references therein). Both the WISE W1-band geometric albedo $p_{W 1}$ and the PTF-derived bond albedo ${ }^{8} A_{\text {bond }}$ show evidence of bimodality among objects with color indices less than 0.25 (Figure 3 panel (D)). Thus, we divide the $\mathrm{C}$ types into low-bond-albedo $\left(\mathrm{C}_{\text {low }}\right)$ and high-bond-albedo $\left(\mathrm{C}_{\text {high }}\right)$ subgroups based on their $p_{W 1}$ as reported by Masiero et al. (2014). In Section 6 we show that the $C_{\text {high }}$ types most closely correspond to what other authors have called $\mathrm{M}$ types.

Waszczak et al. (2015) computed $A_{\text {bond }}$ and $G_{12}$ values for $\sim 1600$ asteroid lightcurves in the PTF survey. Using that work's data (Figure 3 ) we compute median $A_{\text {bond }}$ and $G_{12}$ values (and associated scatter) for the $\mathrm{S}, \mathrm{C}_{\text {low }}$ and $\mathrm{C}_{\text {high }}$ taxonomic groups. Table 2 summarizes the definitions and

\footnotetext{
6 Similar to the color data, the diameter data set we use is a compilation of products from several surveys and described in the appendix of Waszczak et al. (2015). The source IR surveys are WISE (Wright et al. 2010; Masiero et al. 2011, 2014), IRAS (Matson et al. 1986; Tedesco et al. 2002), MSX (Tedesco et al. 2002), and AKARI (Usui et al. 2011).

7 Equation (4) follows directly from combining Equations (1)-(3) The constant $1329 \mathrm{~km}$ depends on somewhat arbitrarily defined quantities such as the Sun's visual magnitude and the ratio of an AU to a kilometer.

8 The visible bond albedo $A_{\text {bond }}$ uses the same WISE diameter used by Masiero et al. (2014) in computing the W1-band geometric albedo $p_{W 1}$.
}

Table 2

$A_{\text {bond }}$ and $G_{12}$ (Based on PTF Data) of Color-defined Taxonomic Groups

\begin{tabular}{lcccccc}
\hline \hline Class & Color & WISE & $\begin{array}{c}A_{\text {bond }} \\
\text { Median }\end{array}$ & $\begin{array}{c}A_{\text {bond }} \\
\text { Scatter }^{\mathrm{a}}\end{array}$ & $\begin{array}{c}G_{12} \\
\text { Median }\end{array}$ & $\begin{array}{c}G_{12} \\
\text { Scatter }^{\mathrm{a}}\end{array}$ \\
\hline $\mathrm{S}$ & $>0.75$ & N.A. & 0.056 & 0.016 & 0.36 & 0.16 \\
$\mathrm{C}_{\text {high }}$ & $<0.25$ & $>0.125$ & 0.038 & 0.022 & 0.42 & 0.20 \\
$\mathrm{C}_{\text {low }}$ & $<0.25$ & $<0.125$ & 0.010 & 0.003 & 0.84 & 0.16 \\
\hline
\end{tabular}

${ }^{\mathrm{a}}$ Scatter is defined here as $0.5 \times(84$ th percentile -16 th percentile $)$.

assumed $A_{\text {bond }}$ and $G_{12}$ values of these groups. There are 245 GALEX-observed asteroids (out of the 405 in Table 1) which have color and diameter data available, allowing them to be modeled by this method. To each GALEX-observed asteroid we assign the appropriate $A_{\text {bond }}$ and $G_{12}$ value based on its class membership, then use its diameter to compute a model absolute magnitude $\left(H_{\mathrm{PTF}}\right)$ using Equation (4). Together with the assumed $G_{12}$ value, this $H_{\mathrm{PTF}}$ then predicts the apparent magnitude $V_{\mathrm{PTF}}$ at each GALEX-observed solar phase angle.

\subsection{Rotational Uncertainty in $V$}

Both the $V_{\mathrm{MPC}}$ and $V_{\mathrm{PTF}}$ model magnitudes discussed here lack an estimate of the rotational term ( $\delta$ in Equation (1)). We account for this by incorporating a term for rotational modulation into the reported uncertainty of $V$. Of the 315 asteroids with $V_{\text {MPC }}$ values, 302 have an amplitude lower limit listed in the Lightcurve Database (Warner et al. 2009; Harris et al. 2012), while for the 245 asteroids with $V_{\text {PTF }}$ predictions there are 239 with reported amplitudes. As shown for instance by Waszczak et al. (2015), asteroids in the relevant size range typically have amplitudes less than $\sim 0.4 \mathrm{mag}$. For the few objects in our sample lacking an amplitude limit, we assume a value of $0.2 \mathrm{mag}$.

Assuming an asteroid's rotational phase $\phi$ to be random at the time of a GALEX detection (i.e., with a probability distribution of the form $P(\phi) \propto$ constant), then the probability distribution of a basic sinusoidal $\delta$ (i.e., one of the form $\delta=\delta_{0} \sin \varphi$ ) can be shown to have the form

$$
P(\delta) \propto \frac{1}{\sqrt{\delta_{0}^{2}-\delta^{2}}},
$$

where $\delta_{0}$ is the amplitude. We use Equation (6) as a probability density function to generate, for each modeled $V$, a set of $10^{4}$ 

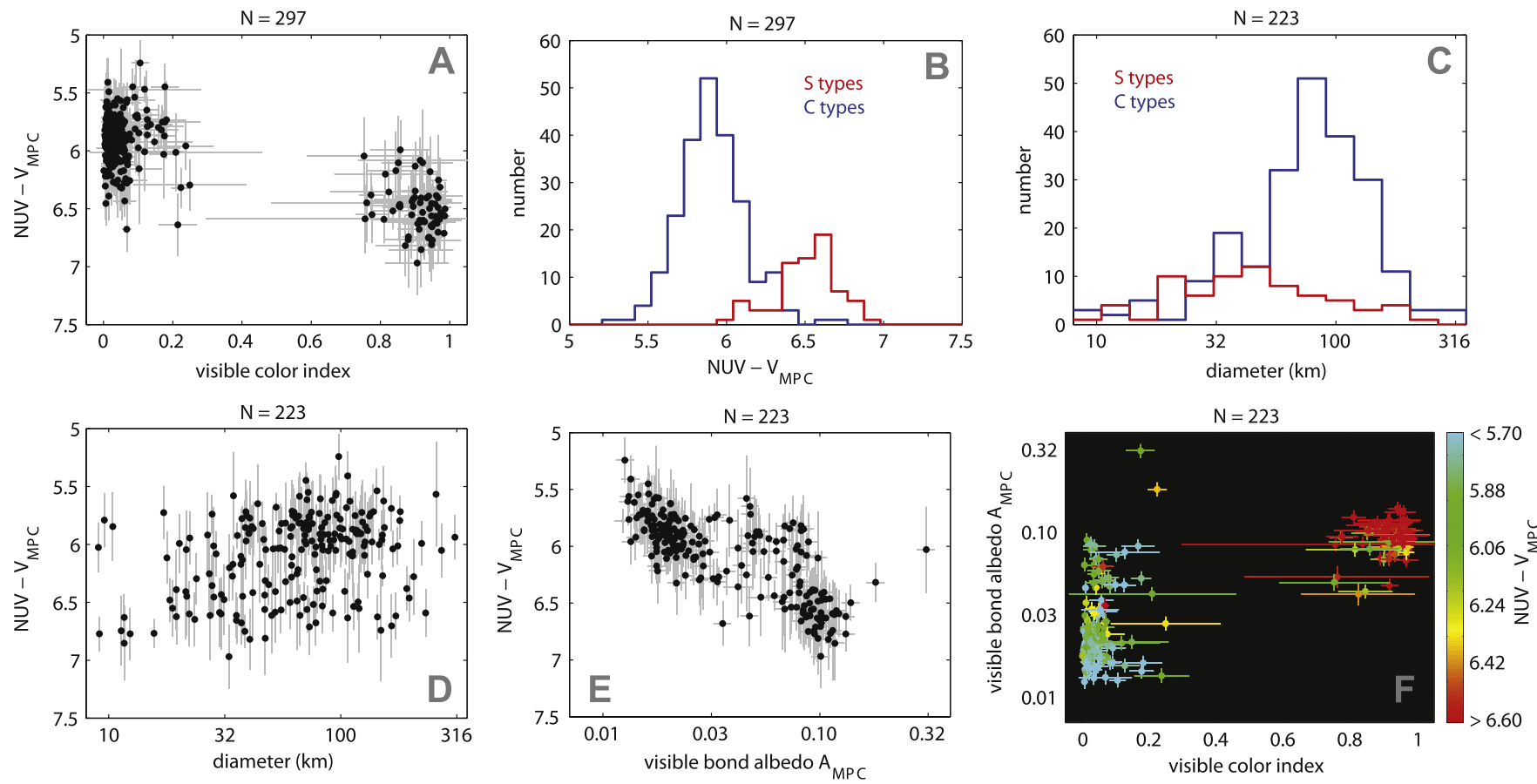

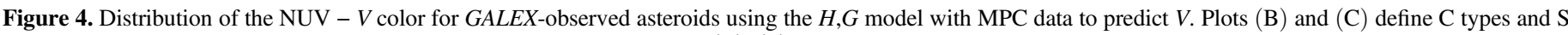

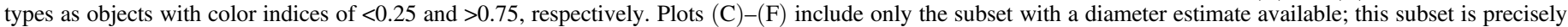
the same sample considered in Figure 6.

simulated $\delta$ values. These simulated $\delta$ are added to an equal number of model $V$ magnitudes computed by random (Gaussian distribution) sampling of the component terms: in the case of $V_{\text {MPC }}$ we just assume a fixed $H_{\text {MPC }}$ uncertainty of $0.1 \mathrm{mag}$, whereas for the $V_{\mathrm{PTF}}$ values we randomly sample all three of $A_{\text {bond }}, G_{12}$, and $D$, using the scatter values in Table 2 for the first two and the literature-reported diameter uncertainty for $D$. The 16th to 84th percentile spread in the distribution of combined $\delta+V$ values then becomes the quoted uncertainty for $V$.

\section{NUV - $V$ COLOR DISTRIBUTION}

Having computed the model $V$ magnitudes, we obtain the NUV - $V$ color for each GALEX asteroid detection and the corresponding uncertainty. The latter contains an additional rotational uncertainty component (now associated with the NUV observation), again determined by repeated sampling of Equation (4) as described above. Since all the asteroids we consider have more than one GALEX NUV detection, we compute the inverse-variance-weighted average NUV $-V$ color for each asteroid (plotted in Figures 4 and 6); the uncertainty in this average is the inverse quadrature sum of the individual uncertainties.

In Figures 4 and 6 (and the accompanying analysis) we have omitted all asteroids with NUV - $V$ uncertainties of greater than $0.5 \mathrm{mag}$. As a result, the sample size of asteroids with

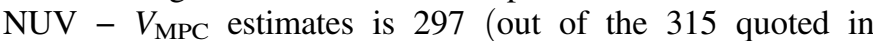
Section 3.1), while the sample with NUV $-V_{\mathrm{PTF}}$ estimates is 223 (out of the 245 quoted in Section 3.2). Figure 5 graphically summarizes the sample selection criteria in a flowchart. In Figures 4, 6 and 7, the errorbars on the color indices were computed by a bootstrapping process described in the appendix of Waszczak et al. (2015).

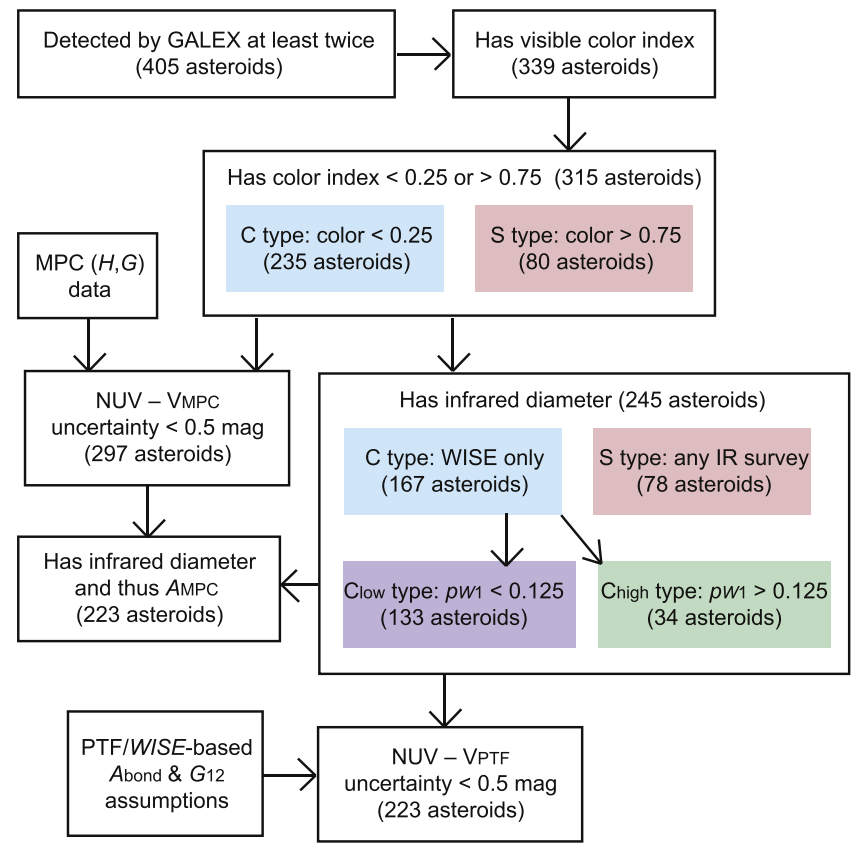

Figure 5. Flowchart visualizing the steps in the $G A L E X$-observed asteroid sample selection process. Each box is a subset of the box pointing to it.

Both the $V_{\mathrm{MPC}}$ and $V_{\mathrm{PTF}}$ model magnitudes produce a bimodal NUV $-V$ color distribution, with the $S$ types having the redder NUV $-V$ color (panels (A) and (B) of both Figures 4 and 6). Median and scatter of NUV $-V$ for the various classes appear in Table 3. To formally ascertain the inequality of the two distributions, we use the two-sided Kolmogorov-Smirnov (KS) test (Massey 1951), which compares two empirical distributions. In particular this test computes a statistic quantifying the extent to which the cumulative distribution function differs in the two 

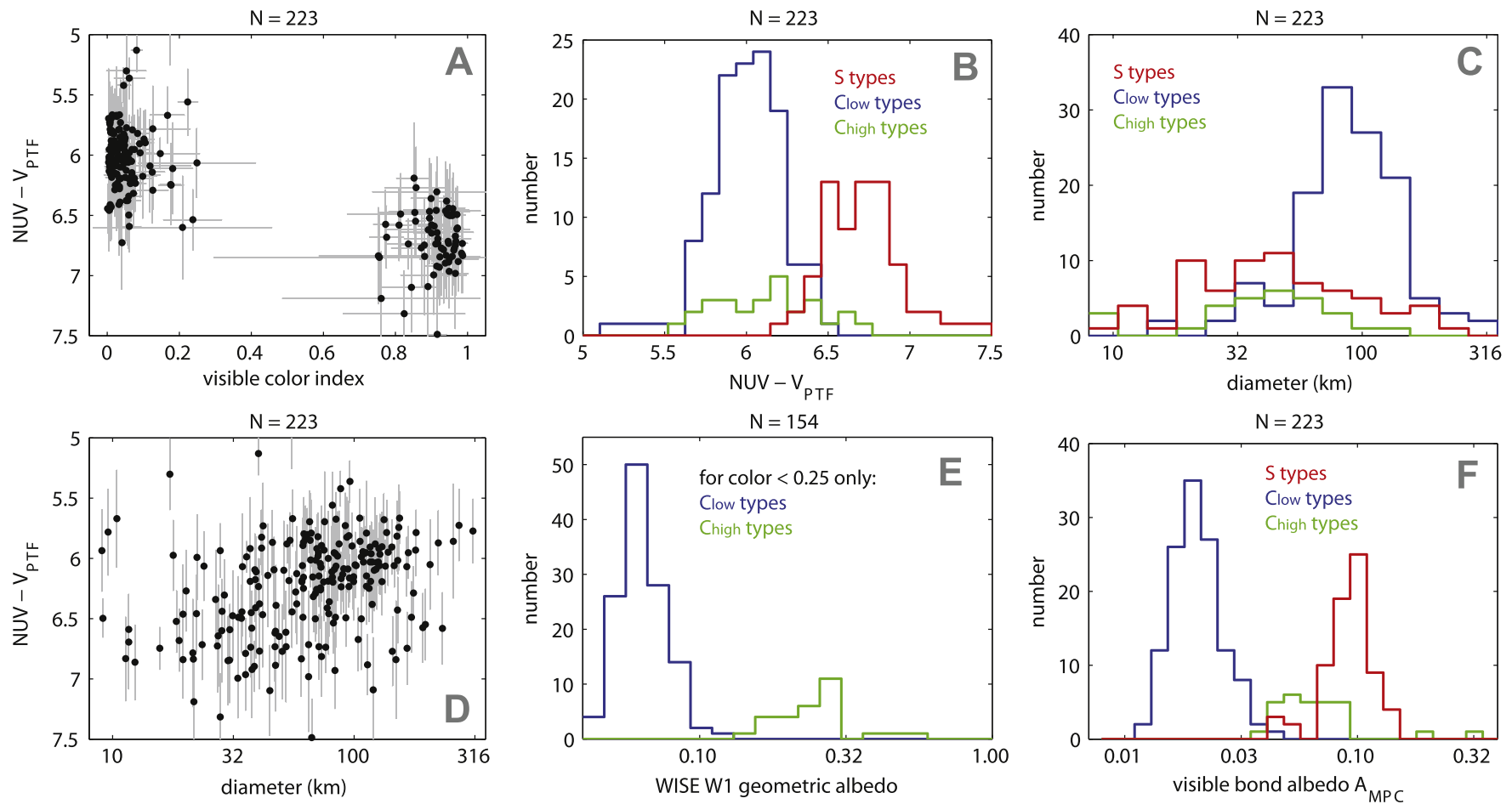

Figure 6. Distribution of the NUV - $V$ color for $G A L E X$-observed asteroids using the $D, A_{\text {bond }}, G_{12}$ model with PTF data, infrared data, and color data to to predict $V$. See Table 2 for the definitions of the $\mathrm{S}, \mathrm{C}_{\text {low }}$, and $\mathrm{C}_{\text {high }}$ groups in plots $(\mathrm{B}),(\mathrm{C}),(\mathrm{E})$, and $(\mathrm{F})$.
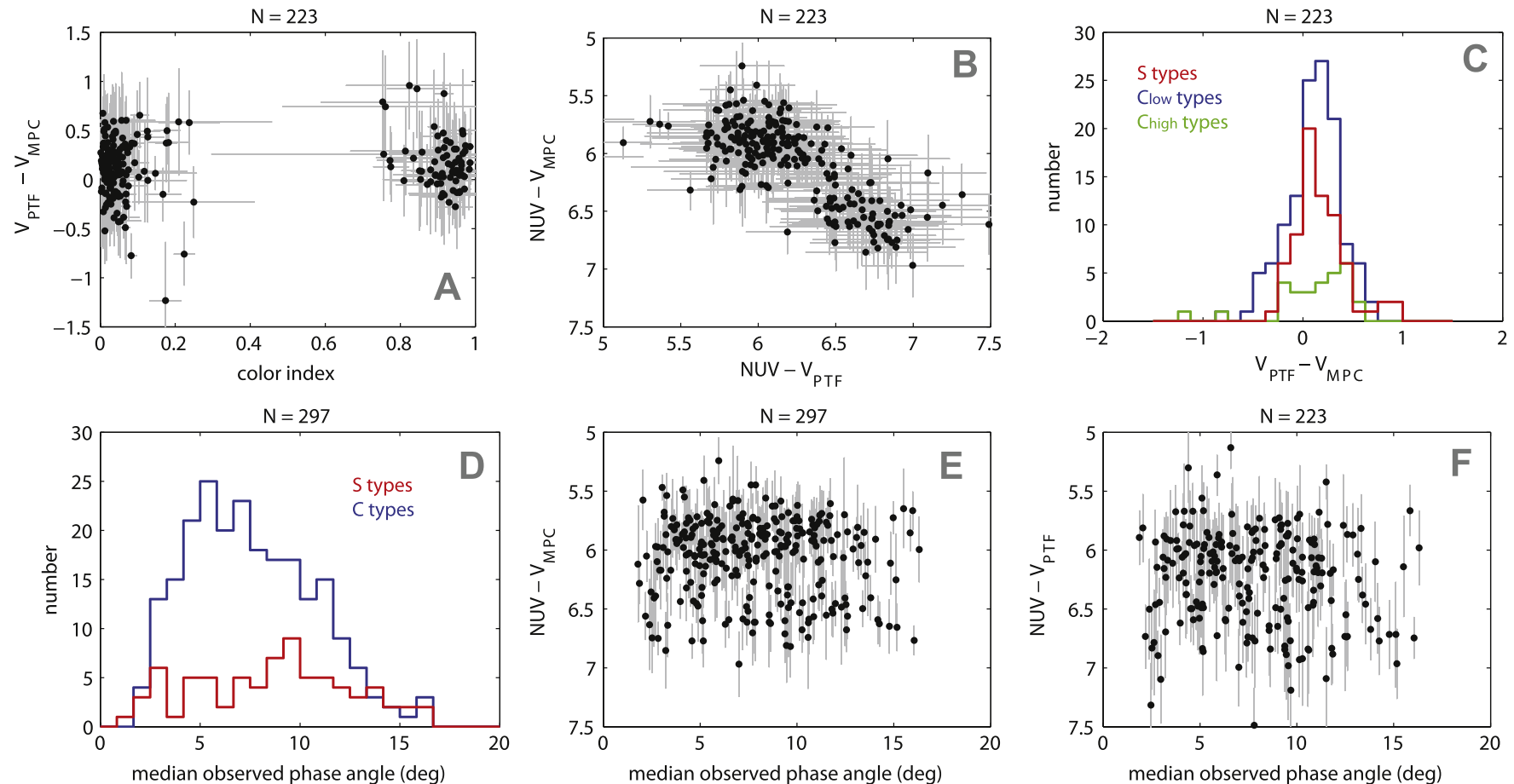

Figure 7. Various checks for systematic differences in the predicted $V$ magnitudes output by the two different photometric models. Bottom row: investigation of phase-angle-dependence on the NUV - $V$ color.

distributions being compared. For the $V_{\mathrm{MPC}}$ model we find the C-type NUV - $V$ color distribution differs from that of the S-type distribution at an $11.6 \sigma$ significance level (Figure 4 panel (B)). For the $V_{\text {PTF }}$ model (Figure 6 panel (B)) we find the $\mathrm{C}$ types $\left(\mathrm{C}_{\text {low }}\right.$ and $\mathrm{C}_{\text {high }}$ combined) differ from the $\mathrm{S}$ types at an $8.1 \sigma$ level, while the $\mathrm{C}_{\text {low }}$ and $\mathrm{C}_{\text {high }}$ types only differ at a $1.9 \sigma$ level (this difference is thus not statistically significant).
An important characteristic of our sample is that the $\mathrm{C}$ types outnumber the $S$ types by a ratio of 3:1 in the $V_{\text {MPC }}$ sample and a ratio of 2:1 in the $V_{\mathrm{PTF}}$ sample (cf. panel (C) of Figures 4 and 6). This ratio is a combination of (1) the inherent difference in the population sizes of the two types (above a given diameter cut-off), a detection bias due to $\mathrm{S}$ types dominating the inner main-belt and thus typically having brighter apparent 
Table 3

NUV - V Color (Mag. Units) of GALEX-observed Asteroids and Sample Sizes

\begin{tabular}{lcccccc}
\hline \hline Class & \multicolumn{2}{c}{ NUV $-V_{\text {MPC }}$} & \multicolumn{2}{c}{ NUV $-V_{\text {PTF }}$} & $N_{\text {MPC }}$ & $N_{\text {PTF }}$ \\
Name & Median & Scatter $^{\text {a }}$ & Median & Scatter $^{\text {a }}$ & & \\
\hline S & 6.52 & 0.25 & 6.71 & 0.21 & 72 & 69 \\
C & 5.90 & 0.19 & 6.03 & 0.22 & 225 & 154 \\
C $_{\text {high }}$ & $\cdots$ & $\cdots$ & 6.14 & 0.33 & $\cdots$ & 29 \\
Clow $_{\text {low }}$ & $\cdots$ & $\cdots$ & 6.02 & 0.19 & $\cdots$ & 125 \\
\hline
\end{tabular}

${ }^{\mathrm{a}}$ Scatter is defined here as $0.5 \times(84$ th percentile -16 th percentile $)$.

magnitudes for a given size and albedo, and (3) the difference in the S and C types' NUV albedo (discussed in Section 5).

In panels $(\mathrm{C})-(\mathrm{F})$ of Figure 4 the sample size decreases from $N=297$ down to $N=223$ asteroids as we consider only those objects in the $V_{\mathrm{MPC}}$ sample that also have available diameters (this is equivalently the $V_{\mathrm{PTF}}$ sample considered in Figure 6). We compute the MPC-based visible bond albedo $A_{\text {MPC }}$ using Equation (2) together with the asteroid's $H_{\mathrm{MPC}}$ and $G_{\mathrm{MPC}}$ values. In particular, there are 38 asteroids (out of the 223 with diameters) with a measured $G_{\mathrm{MPC}} \neq 0.15$; for the remainder we assume $G_{\mathrm{MPC}}=0.15$ for consistency with the manner in which the $V_{\mathrm{MPC}}$ are computed. Analogous to Equation (5), the phase integral for the $G$-model (required for computation of $A_{\mathrm{MPC}}$ via Equation (4)) is

$$
q(G)=0.290+0.684 G
$$

as given by Bowell et al. (1989, pp. 524-556). With the $G_{\mathrm{MPC}}=0.15$ assumption for the majority of the asteroids in our sample, the $A_{\mathrm{MPC}}$ values are not expected to be as accurate as the $A_{\text {bond }}$ values computed for instance by Waszczak et al. (2015), wherein distinct $q$ values were fitted to each object on the basis of a lightcurve. Nonetheless, it is instructive to compute $A_{\mathrm{MPC}}$, e.g., to check for consistency with the classmedian $A_{\text {bond }}$ values, and to exploit as a second taxonomic metric in addition to visible color.

Figure 4 panel (E) shows that NUV $-V$ correlates with $A_{\mathrm{MPC}}\left(\rho_{\text {Spearman }}=0.698,>10 \sigma\right.$ significance $)$, similar to how NUV - $V$ correlates with the color index in panel (A) $\left(\rho_{\text {Spearman }}=0.491,>10 \sigma\right.$ significance $)$. Unlike the color index however, the separation between the $\mathrm{C}_{\text {low }}$ and $\mathrm{C}_{\text {high }}$ subgroups is qualitatively evident in this plot. Figure 4 panel $(\mathrm{F})$ combines all three parameters; note the axes are the same as Figure 3 panel (B), with $A_{\mathrm{MPC}}$ replacing $A_{\text {bond }}$ and the data consisting of GALEX-observed asteroids rather than PTF-observed asteroids.

Figure 6 panel $(\mathrm{F})$ confirms (independently of Figure 3 panel (D)) the validity of using WISE W1-band geometric albedo as a proxy for visible bond albedo to separate $\mathrm{C}_{\text {low }}$ from $\mathrm{C}_{\mathrm{high}}$ - the two classes robustly differ in their $A_{\mathrm{MPC}}$ distributions $(9.5 \sigma$ $\mathrm{KS}$-test significance). However, the class-median $A_{\mathrm{MPC}}$ values of the $\mathrm{C}_{\text {low }}, \mathrm{C}_{\text {high }}$, and $\mathrm{S}$ types are $100 \%, 67 \%$, and $63 \%$ greater than their class-median PTF-based $A_{\text {bond }}$ values in Table 2. This reflects the differing values of $H$ and $q$ produced by the $G$ and $G_{12}$ models, as well as the fact that we apply class-specific $G_{12}$ values, whereas $G_{\mathrm{MPC}}=0.15$ is assumed for the majority of asteroids, regardless of their class.

Consideration of both the $V_{\mathrm{MPC}}$ and $V_{\mathrm{PTF}}$ model magnitudes provides two independent means of computing NUV $-V$; this helps rule out the effect of potential systematic errors unique to either one of the $V$ models, as well as possible biases in the distinct observational data sets upon which each $V$ is based. In
Figure 7 panels $(\mathrm{A})-(\mathrm{C})$ we examine the distribution of $V_{\mathrm{MPC}}$ - $V_{\text {PTF }}$ for all 223 asteroids having both $V$ estimates. The median of $V_{\mathrm{MPC}}-V_{\mathrm{PTF}}$ is $0.13 \mathrm{mag}$ (scatter of $0.25 \mathrm{mag}$ ), indicating the MPC-based model consistently produces brighter $V$ estimates. For $\mathrm{C}$ types the median $V_{\mathrm{MPC}}-V_{\mathrm{PTF}}$ is $0.14 \mathrm{mag}$ and for $\mathrm{S}$ types it is $0.12 \mathrm{mag}$; the two groups' $V_{\mathrm{MPC}}-V_{\mathrm{PTF}}$ distributions differ with less than $0.1 \sigma$ significance in a KS-test.

In Section 1 we motivated our choice to examine the difference in apparent magnitude between UV and visible (as opposed to the difference in albedo in UV and visible) by noting that little is known of asteroid phase functions in the $\mathrm{UV}$, rendering difficult the estimation of UV absolute magnitudes (and hence UV albedos). A potential issue with this approach which we have heretofore ignored is that, if the phase function does differ significantly in the UV from the visible, then the NUV - $V$ color will vary with phase angle. Figure 7 panels $(\mathrm{D})-(\mathrm{F})$ attempt to ascertain whether such a trend exists by considering the median phase angle at which each asteroid was detected by GALEX.

$\mathrm{C}$ types are observed at a median median phase angle of $7: 2$ compared to the $S$ types' median median phase angle of $9: 0$. This is explained by the fact that $\mathrm{C}$ types on average have larger semimajor axes, which geometrically correspond to lower observed phase angles from Earth. Within the C-type group, median phase angle correlates with NUV $-V_{\text {MPC }}$ at $\rho_{\text {Spearman }}=-0.1(1.5 \sigma$ significance $)$ and with NUV $-V_{\mathrm{PTF}}$ at $\rho_{\text {Spearman }}=0.01(0.1 \sigma$ significance $)$. Among $\mathrm{S}$ types, median phase angle correlates with NUV $-V_{\text {MPC }}$ at $\rho_{\text {Spearman }}=0.01$ $(0.1 \sigma$ significance $)$ and with NUV $-V_{\mathrm{PTF}}$ at $\rho_{\text {Spearman }}=0.07$ $(0.5 \sigma$ significance). We therefore cannot claim any phase angle dependence for NUV $-V$, regardless of the taxonomic group or $V$-model being considered.

Various works (e.g., Sanchez et al. 2012 and references therein) discuss the phenomenon of asteroid phase reddening, i.e., an observed reddening of visible color with increasing phase angle. Very few survey-scale samples have been used to test for the presence of this effect. Szabó et al. (2007) computed slightly different phase-angle dependences for the $g-r$ and $r-i$ colors of Trojans in SDSS, though these relations were not separately computed for the Trojans' two taxonomic groups. Waszczak et al. (2015) did not detect any statistically significant difference between $G_{12}$ fits to $r$-band PTF lightcurves and $g$-band PTF lightcurves (among asteroids that had data in both bands). The extent to which a phase-function dependence on wavelength exists between the UV and visible remains unclear. Future UV surveys such as ULTRASAT (Sagiv et al. 2014) offer the most promising means of testing this hypothesis, especially because (unlike GALEX) they will obtain sufficient numbers of observations to adequately sample UV lightcurves, thereby providing the best possible data set for fitting UV phase functions.

\section{ALBEDO VERSUS WAVELENGTH}

If we assume that the phase function does not differ significantly between the UV and visible (or take Figure 7 panels $(\mathrm{E})$ and $(\mathrm{F})$ as justification of this statement), then we can compare the relative bond albedo versus wavelength for the different taxonomic groups using measured colors, filter response functions and the solar spectrum. If the phase function does in fact vary significantly with wavelength, then this approach only provides the relative geometric albedo versus wavelength (see Equations (2) and (3)). 


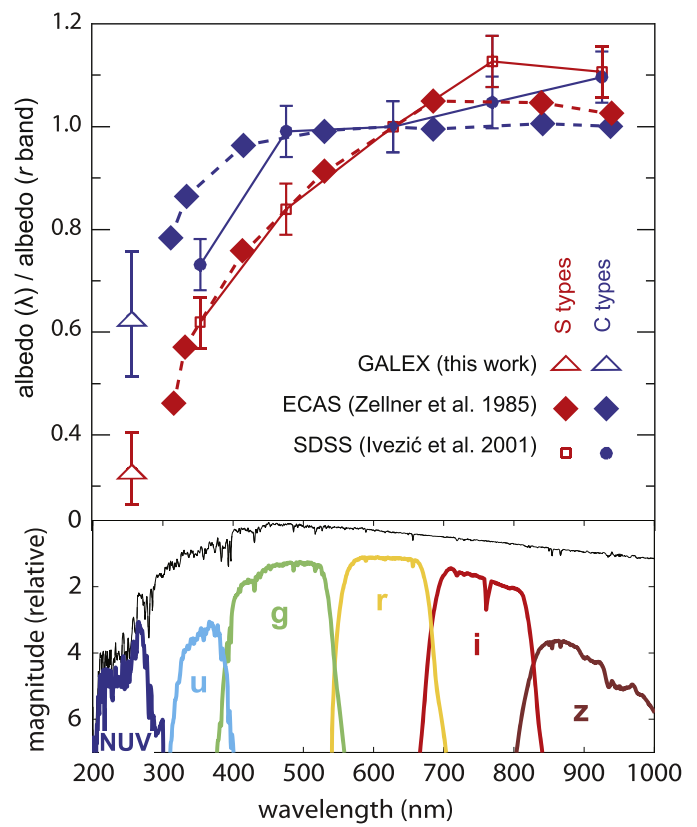

Figure 8. Top: relative albedo (each type's $r$-band albedo normalized to unity) vs. wavelength for $\mathrm{C}$ types and $\mathrm{S}$ types. The leftmost (NUV) points are computed from this work's data, the remaining albedos (SDSS bands) are taken directly from Ivezić et al. (2001). Bottom: bandpass response functions (colored lines) multiplied by the solar spectrum (black line).

Assume photometry from two filters (1 and 2) produce the color measurement $m_{1}-m_{2}$. This color relates to the solar flux distribution $S(\lambda)$, the albedos in each band $\left(A_{1}\right.$ and $\left.A_{2}\right)$ and the filter responses $F_{1}(\lambda)$ and $F_{2}(\lambda)$ according to

$$
10^{\left(m_{1}-m_{2}\right) / 2.5}=\frac{\int F_{1}(\lambda) \lambda^{-2} d \lambda \int S(\lambda) F_{2}(\lambda) A_{2} d \lambda}{\int S(\lambda) F_{1}(\lambda) A_{1} d \lambda \int F_{2}(\lambda) \lambda^{-2} d \lambda}
$$

which we adapted from a similar equation in Pickles (1998).

Using NUV as band 1 and $V$ as band 2 in Equation (6), we use the colors in Table 3 (specifically, the $V_{\mathrm{PTF}}$-based colors) to obtain the albedo ratio $A_{\mathrm{NUV}} / A_{V}$, with uncertainties coming from the associated scatter in the colors. In Figure 7 we plot these albedo ratios for the $\mathrm{C}$ types and $\mathrm{S}$ types, incorporating an additional uncertainty component from the transformation from $r$ to $V$ (see Waszczak et al. 2015 for a discussion of this transformation in the context of asteroids). The end-computed values are $\left(A_{\mathrm{NUV}} / A_{r}\right)_{\mathrm{C}}=0.63_{-0.12}^{+0.14}$ and $\left(A_{\mathrm{NUV}} / A_{r}\right)_{\mathrm{S}}=$ $0.33_{-0.06}^{+0.07}$. The relative albedo values in the SDSS bands included for comparison in Figure 8 are taken directly from a figure in Ivezić et al. (2001); likewise the ECAS data are taken directly from a figure in Zellner et al. (1998).

Note that Figures 8 and 9 ignore the fact that the $S$ types' and C types' absolute albedo in $r$-band differs. In other words, these plots could be converted into ones with absolute albedo on the vertical scale by multiplying the blue and red lines by their respective absolute $r$-band albedos, which would be similar to those listed in Table 2 for $V$ band. Assuming (from Table 2) that $\mathrm{C}$ types have $p_{V}=0.054 \pm 0.019$, then $\left(p_{\mathrm{NUV}}\right)_{\mathrm{C}}=0.035 \pm 0.019$, and assuming $\mathrm{S}$ types have $p_{V}=0.264 \pm 0.031$, then $\left(A_{\mathrm{NUV}}\right)_{\mathrm{S}}=0.088 \pm 0.028$. That is, $\mathrm{S}$ types have a higher mean NUV albedo than $\mathrm{C}$ types, similar to the visible.

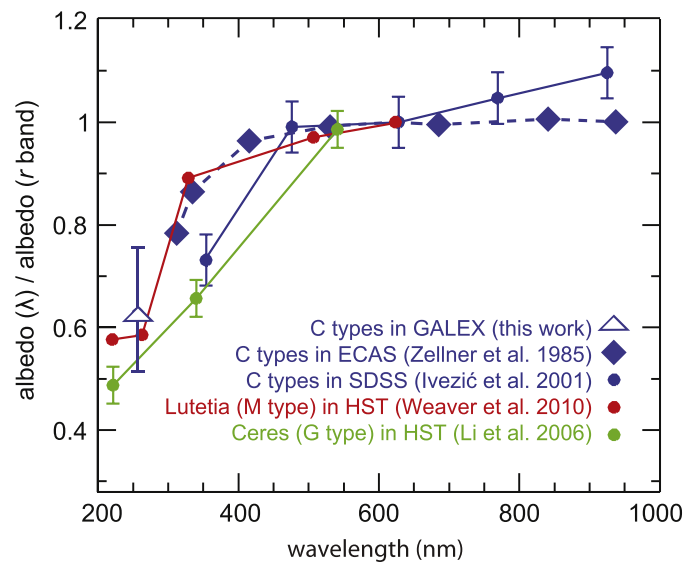

Figure 9. Top: GALEX, SDSS, and ECAS C-type data from Figure 8 compared to HST-derived albedos for 1 Ceres and 21 Lutetia. Note the red color here has a different meaning than it does in Figure 8.

Both $\mathrm{C}$ and $\mathrm{S}$ types show a continued trend of decreasing albedo at shorter wavelengths. Whereas for $\mathrm{S}$ types this behavior was already well-established in the $300-800 \mathrm{~nm}$ region, for $\mathrm{C}$ types the $u-g$ color had previously represented a significant deviation from the shallower slope observed from $400-800 \mathrm{~nm}$. The C-type NUV albedo in Figure 8 confirms the presence of a marked drop in albedo somewhere in the $200-400 \mathrm{~nm}$ range. Given the resolution of Figure 8 and the uncertainties in the data points, we cannot judge whether the C-type albedo levels off between NUV and $u$ band, or whether the slope between $u$ and $g$ bands persists into these shorter wavelengths. The IUE spectra from Roettger \& Buratti (1994) indicate C-type albedo is constant at least in the range 240-300 nm (as does the Lutetia data described below), so that the former interpretation may be more accurate.

\section{COMPARISON TO HST DATA}

\subsection{Lutetia}

Weaver et al. (2010) obtained HST photometry of asteroid 21 Lutetia in UV and visible bands. Lutetia has been classified by various authors as an M-type asteroid; in the context of this work its color index is 0.05 (making it a $\mathrm{C}$ type) and its $A_{\mathrm{MPC}}=0.06$ suggest it to be a $\mathrm{C}_{\text {high }}$ type in particular, though in this work's system we formally would require a WISE $p_{W 1}$ measurement to classify it as such. In the following section we show that $M$ types (a group in the Tholen taxonomic system) and our $\mathrm{C}_{\text {high }}$ types are largely the same population.

The HST Lutetia photometry revealed a steep drop in albedo around $\sim 300 \mathrm{~nm}$ and nearly constant albedo in the 200-300 nm region at a factor $\sim 0.6$ times the visible ( $r$-band-equivalent) albedo. The HST observations of Lutetia thus generally agree with the C-type albedo trend (Figure 9), the main difference being the location of the UV albedo drop-off (the bluest two ECAS bands also demonstrate this difference between $M$ types and C types, e.g., see Figure 2 of Bus et al. (2002, pp. 169-182). The Rosetta spacecraft's flyby of Lutetia enabled FUV observations with the on-board Alice UV imaging spectrograph (Stern et al. 2011); the longest wavelengths of the FUV data $(\sim 190 \mathrm{~nm})$ yield an albedo consistent with the constant value measured in the $200-300 \mathrm{~nm}$ range by $H S T$, namely $\sim 0.6$ times that of the visible albedo. 


\subsection{Ceres}

HST photometry of asteroid 1 Ceres has also been obtained in the UV and visible (Parker et al. 2002; Li et al. 2006). With a color index of 0.01 , Ceres is also a $\mathrm{C}$ type in our classification scheme, though its $A_{\mathrm{MPC}}=0.033$ makes its placement in our $\mathrm{C}_{\text {low }}$ versus $\mathrm{C}_{\text {high }}$ groups ambiguous (see Figure 6 panel $(\mathrm{F})$ ). Like Lutetia, Ceres lacks a reported $p_{W 1}$ so that we cannot formally classify it as either $\mathrm{C}_{\text {low }}$ or $\mathrm{C}_{\text {high }}$.

Ceres was observed by GALEX and thus is included in our MPC-data-based analysis; our measured NUV $-V_{\text {MPC }}=6.45$ \pm 0.19 for Ceres make it a clear outlier from the C-type NUV $V_{\text {MPC }}$ distribution (Figure 4 panel (B)). In the Tholen taxonomic system Ceres is classified as a $G$ type; in the following section we show that other $\mathrm{G}$ types exhibit similarly high NUV - $V_{\mathrm{MPC}}$ values but less anomalous NUV $-V_{\mathrm{PTF}}$.

The Parker et al. (2002) HST data show that around $\sim 300 \mathrm{~nm}$ Ceres' albedo drops to as low as $\sim 0.3$ times the visible-band albedo-compared to the factor of $\sim 0.6$ seen for GALEX C types and the Lutetia data-but that around $\sim 200 \mathrm{~nm}$ it appears to rise again to a more typical C-type UV albedo. Roettger \& Buratti (1994) did not observe this unusually deep absorption feature near $300 \mathrm{~nm}$ in their IUE spectrum of Ceres; if real this feature could partially explain the anomalous NUV $V_{\text {MPC }}$ we observe for $\mathrm{G}$ types in GALEX. Figure 9 shows Ceres data in the three HST bands observed by Li et al. (2006), none of which sample the $300 \mathrm{~nm}$ region containing the putative absorption band, though these three bands do generally match the GALEX C-type data.

\section{C-TYPE SUBGROUPS}

C types deserve further consideration for several reasons: (1) $\mathrm{C}$ types outnumber $\mathrm{S}$ types in the GALEX samples by a factor of several, (2) our division of $\mathrm{C}$ types into $\mathrm{C}_{\text {low }}$ and $\mathrm{C}_{\text {high }}$ merits interpretation in more conventional taxonomic systems, and (3) both of the $H S T$-observed asteroids in the previous section are known members of C-type subgroups, the UV properties of which are worth confirming with additional group members.

Figures 10 and 11 detail the distribution of GALEX-observed asteroids belonging to six classes each from the Tholen and Bus/Binzel taxonomic systems (Tholen 1989, pp. 1139-1150; Bus \& Binzel 2002), the latter is sometimes referred to as the SMASSII system after the survey data with which it was derived. These two classification systems were created on the basis of different visible-band color data; a comparison of their group definitions is given in Table 1 of Bus et al. (2002, pp. 169-182). We consider only the subset of GALEX-observed asteroids having both $V_{\mathrm{MPC}}$ and $V_{\mathrm{PTF}}$ model magnitudes and omit subgroups containing less than three objects. In the following subsections we briefly comment on these subgroups.

One key interpretation of these data-supported also by the HST data in Figure 9-is that NUV-band albedo is not very useful for discriminating C-type subgroups, e.g., $\mathrm{M}$ types versus $\mathrm{G}$ types, whereas $u$ band appears to be more diagnostic in this regard. The $u$-band discrepancy between these subgroups was remarked most notably by Zellner et al. (1998) in the ECAS data, but it was unknown at that time (indeed, up until now) whether the discrepancy in UV albedo became more or less pronounced shortward of $\sim 300 \mathrm{~nm}$. The NUV data indicate that the discrepancy lessens in the NUV, as M types do in fact exhibit a step-down in albedo (between NUV and $u$ bands), similar to the step down the $\mathrm{G}$ types exhibit within $u$ band.

\subsection{Complex}

The Tholen system's X-type group includes asteroids with relatively flat visible color, including no substantial absorption in the blue (in contrast to, e.g., the $u$-band drop-off seen in $\mathrm{G}$ types). The subgroups within the $\mathrm{X}$ group include $\mathrm{E}, \mathrm{M}$, and $\mathrm{P}$ types and are distinguishable only by albedo.

The twelve $\mathrm{M}$ types in our sample all have $A_{\mathrm{MPC}}>0.03$ and $p_{W 1}>0.125$, the latter formally makes them all $\mathrm{C}_{\text {high }}$ types in this work's classification system. The M types have NUV $V_{\mathrm{MPC}}=5.89 \pm 0.15$ and NUV $-V_{\mathrm{PTF}}=6.12 \pm 0.21$, neither of which significantly differ from the $\mathrm{C}$-type averages given in Table 3. This is consistent with the above-noted observation that M-type Lutetia's NUV $-V$ is similar to that of the GALEX $\mathrm{C}$ types, despite an obvious difference in $u$-band (Figure 9). Assuming all 29 of the $\mathrm{C}_{\text {high }}$ types in the GALEX sample are in fact $\mathrm{M}$ types, then the $\mathrm{C}_{\text {high }}$ types' slightly higher NUV $V_{\mathrm{PTF}}=6.14 \pm 0.33$ (compared to NUV $-V_{\mathrm{PTF}}=6.03 \pm 0.22$ for the whole $\mathrm{C}$ type group) agrees well with the $\mathrm{M}$ types' slightly higher average.

Complementary to the $\mathrm{M}$ types, the $11 \mathrm{P}$ types in our sample all have $A_{\mathrm{MPC}}<0.03$ and $p_{W 1}<0.125$, the latter formally makes them all $\mathrm{C}_{\text {low }}$ types. The $\mathrm{P}$ types have NUV $V_{\mathrm{MPC}}=5.74 \pm 0.17$ and $\mathrm{NUV}-V_{\mathrm{PTF}}=5.91 \pm 0.13$. These values are less than both models' C-type averages as well as less than the $C_{\text {low }}$ average, suggesting our $C_{\text {low }}$ group includes more diverse objects than just $\mathrm{P}$ types (e.g., the five $\mathrm{F}$ types also all have $A_{\mathrm{MPC}}$ consistent with $\mathrm{C}_{\text {low }}$ ).

There are 14 GALEX-observed asteroids listed simply as $\mathrm{X}$ types in the Tholen system (presumably because no visible albedo was available at the time of classification); Figure 10 shows that these are in fact distributed across both the $\mathrm{C}_{\text {low }}$ and $\mathrm{C}_{\text {high }}$ albedo ranges.

In the Bus/Binzel system, the $\mathrm{X}$ complex consists of four subgroups: $\mathrm{Xc}, \mathrm{Xk}, \mathrm{X}$ and $\mathrm{Xe}$, these being differentiated by their spectral slope and presence of various absorption features. In the GALEX sample the most numerous of these are the $\mathrm{Xc}$ types, which have the least red visible color and seem to include both high and low visible albedo members. Both the Xe and $\mathrm{Xk}$ types have higher visible color indices (with larger uncertainties in the color). As with the Tholen $\mathrm{X}$ types, we see no systematic trends with respect to the NUV properties of these subgroups.

\subsection{G Types}

Three $G A L E X$-observed asteroids are categorized as $\mathrm{G}$ types. Like G-type Ceres, these have intermediate $A_{\mathrm{MPC}}$ and an above-average NUV $-V_{\text {MPC }}=6.22 \pm 0.11$. In contrast, however, the G-type NUV $-V_{\mathrm{PTF}}=5.79 \pm 0.10$ lies slightly below the C-type average. The reason for this discrepancy is that all three $\mathrm{G}$ types in this sample have $p_{W 1}<0.125$ and so are formally classed as $\mathrm{C}_{\text {low }}$ objects, as a result their assumed $A_{\text {bond }}=0.01$ in the computation of $V_{\mathrm{PTF}}$ may be too low. On the other hand, Oszkiewicz et al. (2011) fit $G_{12}=0.88 \pm 0.2$ to Ceres' phase function, suggesting that the assumed $G_{12}=0.84$ \pm 0.14 for $\mathrm{C}_{\text {low }}$ types (Table 2 ) is a more valid assumption for $\mathrm{G}$ types than the $\mathrm{C}_{\text {high }}$ value of $G_{12}=0.42 \pm 0.20$. Hence the $\mathrm{G}$ types seem not to fit well into either of our $\mathrm{C}_{\text {low }}$ or $\mathrm{C}_{\text {high }}$ groups, and hence are not accurately modeled by our $V_{\mathrm{PTF}}$.

The three-asteroid G-type sample's higher than average NUV - $V_{\text {MPC }}$ agrees with the Cere's HST data (Figure 9), which as discussed above could be indicative of an absorption 

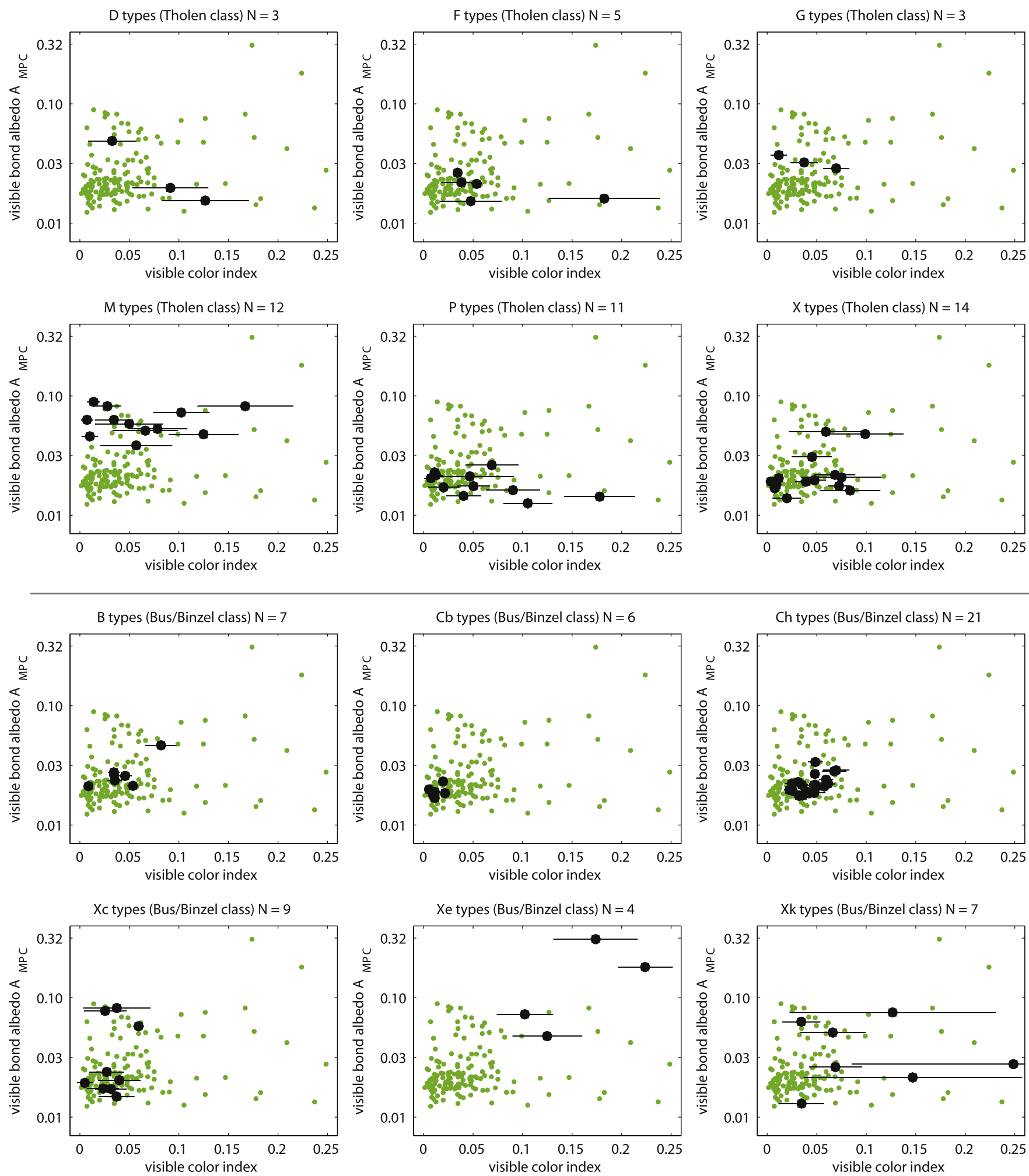

Figure 10. Visible color/albedo distributions of Tholen-classified (Tholen 1989) and Bus/Binzel-classified (Bus \& Binzel 2002) C-type subgroups among this work's sample of GALEX-observed C-type asteroids. See text for further information.

feature at $\sim 300 \mathrm{~nm}$ unique to $\mathrm{G}$ types ( $\mathrm{Li}$ et al. 2006), the precise shape and location of which remains unresolved in the broadband photometry considered here.

Tholen's $\mathrm{G}$ types are represented in the Bus/Binzel system by the $\mathrm{Cg}$ and Cgh groups; however no asteroids in our GALEX sample have either of these SMASSII labels.

\subsection{B Types}

Members of the Tholen B and $\mathrm{F}$ classes, represented also by the Bus/Binzel B and $\mathrm{Cb}$ classes, all are classified as $\mathrm{C}_{\text {low }}$ types in the GALEX sample based on their $p_{W 1}$. Unlike the $\mathrm{G}$ types, the $\mathrm{B}$ types are not anomalous in NUV $-V_{\mathrm{MPC}}$, meaning the $\mathrm{B}$ 

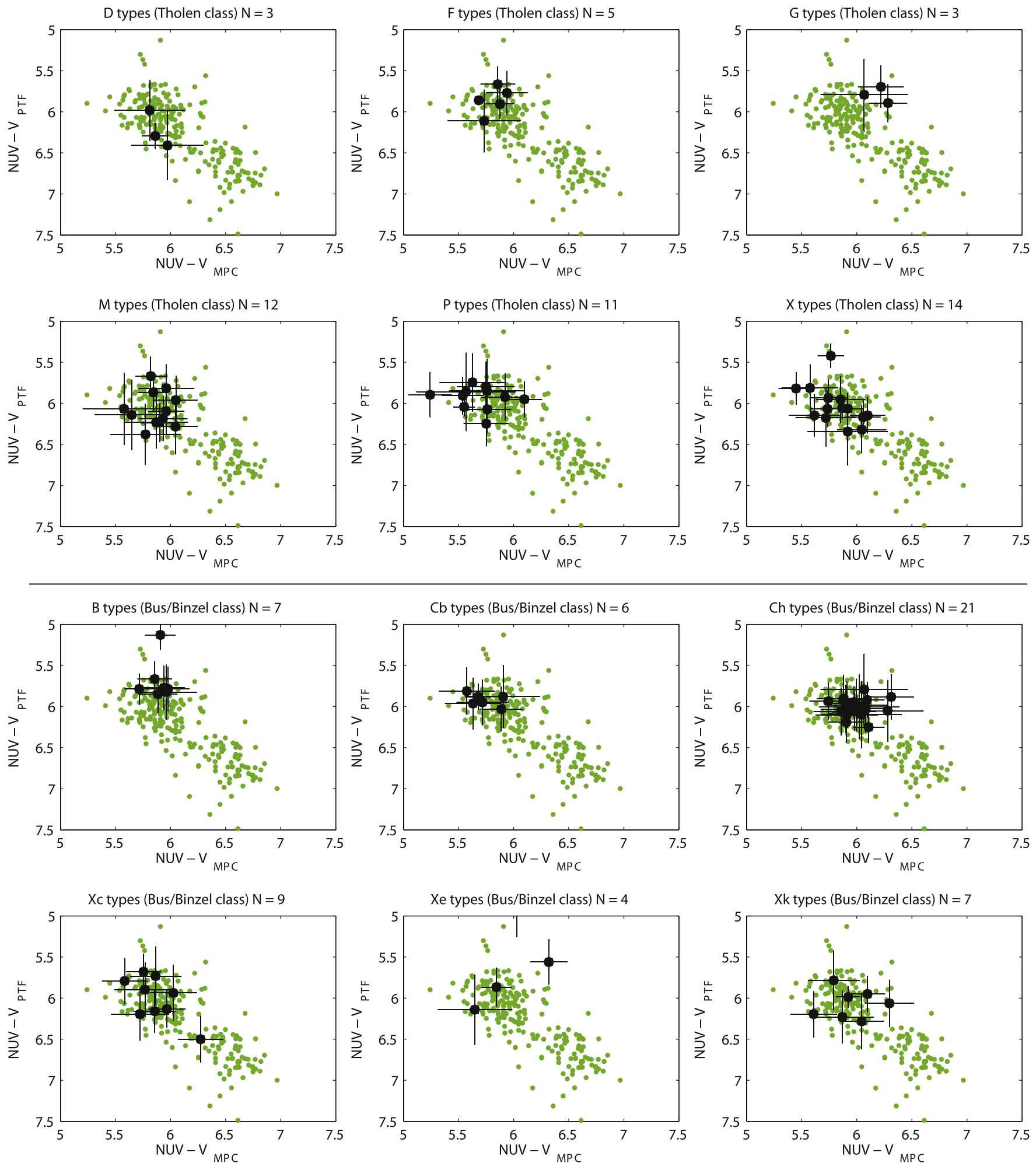

Figure 11. NUV - $V$ color distributions of Tholen-classified (Tholen 1989) and Bus/Binzel-classified (Bus \& Binzel 2002) C-type subgroups among this work's sample of GALEX-observed C-type asteroids. See text for further information.

types likely lack the $\mathrm{G}$ types' strong absorption at $300 \mathrm{~nm}$. The $\mathrm{B}$ types also are characterized by slightly higher $A_{\mathrm{MPC}}=0.026$ compared to the $\mathrm{C}_{\text {low }}$ average $A_{\mathrm{MPC}}=0.020$. Hence, like the $\mathrm{G}$ types, the B types show a lower than average NUV - $V_{\text {PTF }}$ symptomatic of an underestimated $A_{\text {bond }}$ and therefore too dim of a predicted $V_{\text {PTF }}$.

\section{SUMMARY}

We present NUV-band photometry of 405 asteroids observed serendipitously by GALEX from 2003 to 2012. Using a compilation of visible-band color data, we select the subset of these GALEX-observed asteroids belonging to the $\mathrm{C}$-type or $\mathrm{S}$-type classes. We then compute the visual-band magnitude 
Table 4

Glossary of Acronyms and Symbols Used Repeatedly in This Work

\begin{tabular}{|c|c|}
\hline Acronym & Full Meaning/Description \\
\hline$\alpha$ & solar phase angle (angle drawn by a light ray as it travels from the Sun to an asteroid to the Earth) \\
\hline$A_{\text {bond }}$ & visible-band bond albedo \\
\hline$A_{\mathrm{MPC}}$ & visible-band bond albedo computed using $H$ and $G$ values from the MPC together with an infrared-derived diameter \\
\hline$A_{\mathrm{NUV}}$ & near-ultraviolet-band bond albedo \\
\hline $\mathrm{C}_{\text {high }}$ types & C-type asteroids with a high near-infrared albedo $\left(p_{W 1}>0.125\right)$, a group consisting almost exclusively of $\mathrm{M}$ types \\
\hline $\mathrm{C}_{\text {low }}$ types & C-type asteroids with a low near-infrared albedo $\left(p_{W 1}<0.125\right)$, a group consisting of $\mathrm{P}$ types and many $\mathrm{X}$ types \\
\hline$D$ & asteroid diameter \\
\hline ECAS & Eight-Color Asteroid Survey \\
\hline FUV & far-ultraviolet band $(\lambda \lesssim 180 \mathrm{~nm})$ \\
\hline G & an older photometric phase-function model parameter (Bowell et al. 1989) \\
\hline$G_{12}$ & a newer photometric phase-function model parameter (Muinonen et al. 2010) \\
\hline G types & certain asteroids, including Ceres, that are a subgroup of the C-type asteroid taxonomic class \\
\hline GALEX & Galaxy Evolution Explorer satellite \\
\hline$H$ & visible-band absolute magnitude ( $V$ magnitude asteroid would have if observed $1 \mathrm{AU}$ from both the Sun and Earth, at zero phase angle) \\
\hline HST & Hubble Space Telescope \\
\hline IUE & International Ultraviolet Explorer satellite \\
\hline MPC & Minor Planet Center, http://minorplanetcenter.net \\
\hline NUV & near-ultraviolet band $(180-200 \mathrm{~nm})$, and/or measured magnitude in this band \\
\hline PTF & Palomar Transient Factory survey \\
\hline$p_{V}$ & visible-band geometric albedo \\
\hline$p_{W 1}$ & near-infrared (W1-band from WISE) geometric albedo \\
\hline$\rho_{\text {Spearman }}$ & Spearman's correlation coefficient \\
\hline SDSS & Sloan Digital Sky Survey \\
\hline SMASS & Small Main-belt Asteroid Spectroscopic Survey \\
\hline$V$ & visible-band $(\sim 600 \mathrm{~nm})$ astronomical magnitude \\
\hline$V_{\mathrm{MPC}}$ & predicted $V$ magnitude based on MPC-hosted observational data and the $G$ phase-function model \\
\hline$V_{\mathrm{PTF}}$ & predicted $V$ magnitude based on color-class-averaged albedos and phase-functions data derived from PTF data and $G_{12}$ phase-function model \\
\hline$V$ & visible-band $(\sim 600 \mathrm{~nm})$ astronomical magnitude \\
\hline WISE & Wide-field Infrared Explorer satellite \\
\hline
\end{tabular}

(using two different models) corresponding to each GALEX detection in an effort to study the NUV $-V$ color. For both $V$ models, the derived NUV $-V$ color distribution is bimodal, with $\mathrm{S}$ types having the redder color, just as they do within the visible band. The average C-type NUV - V agrees with $H S T$ observations of the asteroids Lutetia and Ceres, both of which are members of the visible-color-defined C-type group. Slight differences in the measured NUV - $V$ among known taxonomic subgroups of the $\mathrm{C}$ types may indicate membership in either the M-type or G-type subgroups, though the $300-400 \mathrm{~nm}$ region ( $u$-band) is more diagnostic of this division.

A. Waszczak has been supported in part by the W.M. Keck Institute for Space Studies (KISS) at Caltech. E.O.O. is incumbent of the Arye Dissentshik career development chair and is grateful to support by grants from the Willner Family Leadership Institute Ilan Gluzman (Secaucus NJ), Israeli Ministry of Science, Israel Science Foundation, Minerva and the I-CORE Program of the Planning and Budgeting Committee and The Israel Science Foundation. This work makes use of data products from the GALEX mission, developed and operated with support from JPL/Caltech, the Centre National d'Etudes Spatiales of France and the Korean Ministry of Science and Technology, Orbital Sciences, the University of California at Berkeley, and the Laboratoire d'Astrophysique de Marseille. This work also makes use of data derived from the Palomar Transient Factory (PTF) and the Intermediate PTF (iPTF) projects, operated at the $1.2 \mathrm{~m}$ Samuel Oschin Telescope at Palomar Observatory.
Participating institutions have included Caltech, Columbia University, Las Cumbres Observatory Global Telescope Network, Lawrence Berkeley National Laboratory, the National Energy Research Scientific Computing Center, the University of Oxford, the Kavli Institute for the Physics and Mathematics of the Universe, Los Alamos National Laboratory, the Oskar Klein Centre, the University System of Taiwan, the University of Wisconsin Milwaukee, and the Weizmann Institute of Science. This work also makes use of data products from the Wide-field Infrared Survey Explorer, which is a joint project of the University of California Los Angeles and the Jet Propulsion Laboratory (JPL)/Caltech, funded by NASA. This publication also makes use of data products from NEOWISE, which is a project of JPL/Caltech, funded by the Planetary Science Division of NASA. This work also makes use of data from the Sloan Digital Sky Survey (SDSS), managed by the Astrophysical Research Consortium for the Participating Institutions and funded by the Alfred P. Sloan Foundation, the Participating Institutions, the National Science Foundation, the US Department of Energy, NASA, the Japanese Monbukagakusho, the Max Planck Society, and the Higher Education Council for England.

\section{Glossary of Acronyms and Symbols}

For the reader's convenience, Table 4 summarizes the various acronyms and mathematical symbols used in this work. 


\section{REFERENCES}

Bowell, E., Hapke, B., Domingue, D., et al. 1989, in Asteroids II, ed. R. P. Binzel, T. Gehrels, \& M. Shapley Matthews (Tucson, AZ: Univ. Arizona Press), 524

Bus, S. J., \& Binzel, R. P. 2002, Icar, 158, 106

Bus, S. J., Vilas, F., \& Barucci, M. 2002, in Asteroids III, ed. W. F. Bottke, Jr. et al. (Tucson, AZ: Univ. Arizona Press), 169

Giorgini, J. D., Yeomans, D. K., Chamberlin, A. B., et al. 1996, BAAS, 28, 1158

Harris, A. W., Warner, B. D., \& Pravec, P. (ed.) 2012, in Asteroid Lightcurve Derived Data V13.0.EAR-A-5-DDR-DERIVED-LIGHTCURVE-V13.0 (NASA Planetary Data System)

Harris, A. W., \& Young, J. W. 1988, BAAS, 20, 865

Ivezić, Ž, Tabachnik, S., Rafikov, R., et al. 2001, AJ, 122, 2749

Law, N. M., Kulkarni, S. R., Dekany, R. G., et al. 2009, PASP, 121, 1395

Li, J. Y., McFadden, L. A., Parker, J. W., et al. 2006, Icar, 182, 143

Li, N., \& Thakar, A. R. 2008, CSE, 10, 18

Martin, D. C., Fanson, J., Schiminovich, D., et al. 2005, AJ, 619, 1

Masiero, J. R., Grav, T., Mainzer, A. K., et al. 2014, AJ, 791, 121

Masiero, J. R., Mainzer, A. K., Grav, T., et al. 2011, AJ, 741, 68

Massey, F. J. 1951, J. Am. Stat. Assoc., 46, 68

Matson, D. L., Veeder, G. J., Tedesco, E. F., Lebofsky, L. A., \& Walker, R. G. 1986, AdSpR, 6, 47

Morrissey, P., Conrow, T., Barlow, T. A., et al. 2007, APJS, 173, 682

Morrissey, P., Schiminovich, D., Barlow, T. A., et al. 2005, APJL, 619, L7

Muinonen, K., Belskaya, I. N., Cellino, A., et al. 2010, Icar, 209, 542

Ofek, E. O. 2012, AJ, 749, 10
Oszkiewicz, D. A., Bowell, E., Wasserman, L. H., et al. 2012, Icarus, 219, 283

Parker, A., Ivezić, Ž, Jurić, M., et al. 2008, Icar, 198, 138

Parker, J. W., Stern, A., Thomas, P. C., et al. 2002, AJ, 123, 549

Pickles, A. J. 1998, PASP, 110, 749

Rau, A., Kulkarni, S. R., Law, N. M., et al. 2009, PASP, 121, 1334

Roettger, E. E., \& Buratti, B. J. 1994, Icar, 112, 496

Sagiv, I., Gal-Yam, A., Ofek, E. O., et al. 2014, AJ, 147, 79

Sanchez, J. A., Reddy, V., Nathues, A., et al. 2012, Icar, 220, 36

Stern, S. A., Parker, J. W., Feldman, P. D., et al. 2011, AJ, 141, 199

Szabó, G. M., Ivezić, Ž, Jurić, M., \& Lupton, R. 2007, MNRAS, 377,1393

Tedesco, E. F., Egan, M. P., \& Price, S. D. 2002, AJ, 124, 583

Tedesco, E. F., Noah, P. V., Noah, M., \& Price, S. D. 2002, AJ, 123, 1056

Tholen, D. J. 1989, in Asteroids II, ed. R. P. Binzel, T. Gehrels, \& M. S. Matthews (Tucson, AZ: Univ. Arizona Press), 1139

Usui, F., Kuroda, D., Müller, T. G., et al. 2011, PASJ, 63, 1117

Wagner, J. K., Hapke, B. W., \& Wells, E. N. 1987, Icar, 69, 14

Warner, B., Harris, A. W., \& Pravec, P. 2009, Icar, 202, 134

Waszczak, A., Chang, C.-K., Ofek, E. O., et al. 2015, arXiv:1504.04041

Waszczak, A., Ofek, E. O., Aharonson, O., et al. 2013, MNRAS, 433, 3115

Weaver, H. A., Feldman, P. D., Merline, W. J., et al. 2010, A\&A, 518, A4

Wright, E. L., Eisenhardt, P. R. M., Mainzer, A. K., et al. 2010, AJ, 140, 1868

Xu, S., Binzel, R. P., Burbine, T. H., \& Bus, S. J. 1995, Icar, 115, 1

York, D. G., Adelman, J., Anderson, J. E., Jr., et al. 2000, AJ, 120, 1579

Zellner, B., Tholen, D. J., \& Tedesco, E. F. 1998, Icar, 61, 355 